\title{
iQbD: a TRL-indexed Quality-by-Design Paradigm for Medical Device Engineering
}

\author{
Thierry Bastogne ${ }^{1}$
}

affiliations: ${ }^{1}$ Université de Lorraine, CNRS, CRAN, F-54000 Nancy, France ${ }^{2}$ INRIA BIGS, F-54000 Nancy, France

abbreviated title: Quality-by-Design for Medical Device Engineering

correspondence: CRAN UL-CNRS UMR 7039

9 av. de la forêt de Haye, Campus Médecine, Bât.D

BP 184

54505 Vandoeuvre-lès-Nancy Cedex

FRANCE

e-mail: thierry.bastogne@univ-lorraine.fr 


\begin{abstract}
Risk assessment is a critical step in the roadmap of medical device development. Failure modes, effects and criticality analysis is a common approach based on declarative prior information that proved beneficial in the risk assessment of well established processes. But at early steps of development when innovative materials or technologies are embedded, the lack of experience on those innovations introduces too much subjectivity in FMECA for a robust risk assessment. Since mid-2000, the Quality-byDesign guideline has been proposed within the pharmaceutical industry as a proactive engineering approach of drug development. This paradigm enables a data-driven risk assessment throughout the development workflow, which completes risk assessment provided by FMECA. Nevertheless, its implementation guide is unclear and not flexible enough to be efficiently applied to the development of medical devices. To address this issue, a new QbD paradigm indexed on the technological readiness level of the innovative product is proposed. It covers the development of medical devices throughout the whole preclinical phase and is composed of at least nine learning cycles. The first part of this medical device QbD layout, composed of three consecutive risk assessment cycles, is evaluated through a real study case with the objective to demonstrate the proof of concept of a photobleaching controller in photodynamic therapy. Beyond this experimental result, this application has confirmed practical ability of the iQbD approach to complete FMECA and to provide an alternative solution to risk assessment when prior knowledge on the technological innovation is not available.
\end{abstract}

keywords: Medical Device, Quality-by-Design, Product Development, Risk Assessment, Regulatory 


\section{Introduction}

The engineering of medical devices is a long and laborious road between the innovative idea and post-market monitoring ${ }^{1}$ where a whole series of risks may cause extensive delays of development. To prevent them as much as possible, a number of studies have been focused on the modeling of the Medical Device Development (MDD) process ${ }^{2}$. $\operatorname{In}^{3}$, a Business Process Model and Notation model is proposed to describe MDD in the European regulatory framework while a Unified Modeling Language model is elaborated in ${ }^{1}$ to analyze the same process in the Food and Drug Administration (FDA) context. Those graphical representations address all aspects involved in MDD: product development, market introduction, regulatory classification, standards and industrial property. Often used in innovation processes, mind maps, a class of learning strategy developed in 70's by T. Buzan ${ }^{4}$, are also used in Medicine ${ }^{5}$ to present complex information concisely and to facilitate the sharing of risk management knowledge. In contrast to those analytic descriptions, Parameter Diagrams (P-diagrams) are holistic models of a product taking the inputs from a system/customer and relates those inputs to desired outputs of a design that the engineer is creating, also considering noncontrollable outside influences ${ }^{6}$. For each of these inputs, the engineer identifies parameters and specifies numerical values to achieve the required output of the final product, and so P-diagrams are relevant tools in brainstorming and documenting input signals, noise factors, control factors, error states and ideal response.

However, all those graphical models cannot alone efficiently address risk assessment, a critical step in the MDD roadmap ${ }^{7}$. That both applies to risks related to efficacy, safety, quality as well as regulatory requirements. The application of risk management to medical devices is essentially governed by the international standard ISO 14971 recently revised in $2019^{8}$. In this standard, risk assessment mainly relies on the application of the failure modes, effects and criticality analysis (FMECA) or equivalent methods detailed in ICH Q99 ${ }^{9}$. In those approaches, a Risk Priority Number (RPN) is computed by evaluating both the severity (S), occurence (O) and detectability (D) levels of hazards according to predefined scoring scales. FMEA has been used as a risk identification and reduction approach for decades. It was 
initially developed by the United States Military in the late 1940s and has been successfully applied for almost 70 years in a wide spectrum of industrial sectors ${ }^{10}$. However, a number of reviews and studies ${ }^{11,12,13}$ have stressed limitations of this method such as the relative importance among $\mathrm{S}, \mathrm{O}$ and $\mathrm{D}$ is not considered; different combinations of $\mathrm{S}, \mathrm{O}$ and $\mathrm{D}$ may produce exactly the same value of RPN, but their hidden risk implications may be totally different; those three risk factors are difficult to be precisely evaluated; the conversion of scores is different for the three risk factors; the RPN cannot be used to measure the effectiveness of corrective actions; interdependencies among various failure modes and effects are not taken into account; etc. $\mathrm{In}^{14,15}$, authors also insist on other weaknesses such as the sensitivity of RPN with respect to the subjectivity/human errors. Indeed, evaluations of S, O and D are based on declarative information provided by developer teams and become productive when participants have gained some experience in the product and its manufacturing process. But at early steps of development or when innovative materials or technologies are embedded, the lack of experience on those innovations introduces too much subjectivity in FMEA for a robust risk assessment and a complementary risk assessment methods is needed.

Several methods are already available to manage and validate quality in industry such as Total Quality Management ${ }^{16}$, Lean Management ${ }^{17}$ and Design for Six-Sigma ${ }^{18}$ in which Design of Experiments (DoE) techniques play a fundamental role. All those techniques belong to the category of Quality by Testing where products are tested only after the design and manufacturing steps. Unfortunately, when problems are detected then, it is usually too late and it can take multiple cycles before getting it right. To anticipate technological risks since the early development steps, J. M. Juran proposed in 1992 the concept of Quality by Design $(\mathrm{QbD})^{19}$.

Since mid-2000, new pharmaceutical development directives have been elaborated by the International Council for Harmonisation of Technical Requirements for Pharmaceuticals for Human Use (ICH) and are detailed in a set of five guidelines, noted Q8 to Q12. The first guideline, ICH Q8, takes up the QbD concept in order to adapt it to today's current challenges within the pharmaceutical industry. Pharmaceutical QbD is a risk-based and 
proactive engineering approach of drug development that begins with predefined objectives and emphasizes product and process understanding, based on sound science and quality risk management ${ }^{20}$. The QbD implementation relies on a large collection of statistical methods and tools essentially devoted to the design of experiments and the multivariate regression analysis. One key differentiation point with previous quality management strategies is the concept of Design Space. The latter is a probabilistic representation of the relationship between between critical quality/safety attributes and formulation/manufacturing factors. It allows developers to identify a region entitled Normal Operating Region in which the probabilities of compliance with quality and safety specifications are acceptable.

In 2015, a FDA study was carried out to assess the applicability of QbD for coating drug eluting stents. A first conclusion was QbD enhanced the understanding about the medical device process development and more particularly its ability to identify rapidly the critical factors affecting its quality attributes. Authors finally concluded that Design Control methodology ${ }^{21}$ and $\mathrm{QbD}$ could certainly benefit from each other. More recently, Martinez-Marquez et al. have presented a successful application of $\mathrm{QbD}$ to the development of 3D printed bone prostheses and scaffolds ${ }^{22,23}$. Since 2019, a European project, entitled TBMED, aims at developing an Open Innovation Test Bed (OITB) for high risk medical devices that provides a single entry point to services along the whole value chain from preclinical development to clinical testing. This OITB will fully rely on the QbD process. However, the reality remains that much work needs to be done in this area. The lack of clarity regarding the different stages of the QbD process can in part explain the small number of applications to medical devices. Moreover, the current QbD paradigm is not flexible enough and does not take into account the technological readiness level into account. In particular, at early steps of development, the QbD implementation can be simplified.

To get a clearer vision of the complete QbD process suited to the development of medical devices but also to address its lack of flexibility, we have proposed an adaptive scheme of QbD indexed to the technological readiness level of the product. To assess its relevance in practice, we have applied the new paradigm to a new medical device devoted to the realtime 
control of the photobleaching trajectory in photodynamic therapy.

This paper is organized as follows. Section 2 starts by setting out the foundations of Quality-by-Design before presenting details of the new adaptive paradigm, the study case and the materials for its implementation. Results are detailed in Section 3 and are discussed in Section 4. Finally, conclusions are drawn in Section 5. Additional outcomes of the study are presented in a supplementary material freely available ${ }^{1}$

\section{Materials and Methods}

\subsection{Conventional Quality-by-Design}

Quality by design (QbD) is an holistic, proactive and integrative approach initially proposed for drug development that begins with predefined objectives and emphasizes product and process understanding, based on sound science and quality risk management. The overarching goal of $\mathrm{QbD}$ is to improve safety and efficacy, while additional benefits include the reduction of cost and the potential for faster regulatory approval of new products. The conventional QbD process is composed of seven main steps as presented in Figure 1.

[Figure 1 about here.]

\subsubsection{Target Product Profile}

The Quality Target Product Profile (QTPP) is a key strategic document that provides summary on the product under development; the product's desired characteristics and features; the studies and activities that must be completed to demonstrate the product efficacy, safety and quality and the features that provide a competitive advantage. Each section of the QTPP provides the opportunity to describe the key differentiating features and competitive positioning of the product to be developed. QTPP is a living document that has to regularly updated throughout the project progress.

\footnotetext{
${ }^{1}$ https://hal .archives-ouvertes.fr/hal-02937273
} 


\subsubsection{CQA Definition and specification}

A critical quality attribute is a physical, chemical or biological property or any other characteristics, which has to be kept within a predefined range to ensure the expected quality specification of the product to be developed. CQA can be regarded as measurable metrics of efficacy/safety or quality objectives.

\subsubsection{CMA and CPP Definition}

Critical manufacturing attributes (CMA) belong to the first category of factors able to cause variability of CQA. They are associated with the formulation parameters of the product. Critical process parameters (CPP) are the second category of potential impacting factors related to the manufacturing parameters. Initially, the concept of critical material attributes did not appear in the ICH-Q8(R2) guideline but was only introduced in the ICH-Q11 concept paper. Unlike CQA, CMA and CPP can be directly modified by the experimenters and are used as technical means to indirectly control CQA values. In terms associated with the control engineering field, CQA can be assimilated with output variables while CMA and $\mathrm{CPP}$ are input variables.

\subsubsection{Design space identification}

A Design Space is the result of a Risk Mapping step. The concept of Design Space relies on a multivariate representation in the $\mathrm{CMA} / \mathrm{CPP}$ space in which at least three regions of interest are identified:

- the Normal Operating Region (NOR) is a subspace in which it is very likely to meet the CQA requirements. A mathematical definition of NOR is given in (2);

- the Proven Acceptance Region (PAR) is another domain in which the probability to get acceptable product is still acceptable, but process adjustments of $\mathrm{CMA} / \mathrm{CPP}$ should be made to return operation to NOR subspace; 
- the Out Of Specifications (OOS) region corresponds to the case where the state of the product is not acceptable. Investigations must take place to determine the reasons and decide on a course of action (control strategy).

$$
\begin{aligned}
\text { NOR } & =\left\{\tilde{x} ; \mathbb{P}\left(\left.\tilde{Y}\right|_{y, x, \tilde{x}} \in \Lambda\right) \geq \rho\right\} \\
& =\left\{\tilde{x} ; \int_{\Lambda} p(\tilde{y} \mid y, x, \tilde{x}) d \tilde{y} \geq \rho\right\}
\end{aligned}
$$

where $\mathbb{P}(\cdot)$ is a probability function, $\tilde{x}$ is a vector of values taken by the critical material attributes and/or critical process parameters. $\{\tilde{x}\}$ denotes the predicted NOR subspace, i.e. a subset of CMA/CPP values for which we have a probability greater than $\rho$ to fulfill the expected performance specifications $\Lambda$ on the predicted CQA: $\tilde{Y}$. The other regions: PAR and OOS are defined by the same equation but with different ranges for $\rho$.

\subsubsection{Process Analytical Technology and Control Strategy}

To implement QbD effectively, we need technology to enable the measurement of CMA/CPP and then to inform about the location of the current product in the design space. This is the role of the Process Analytical Technology (PAT). PAT tools have typically been spectroscopic in nature. Methods such as near-infrared (NIR) and Raman spectroscopy are the most commonly used tools. When a PAT system detects a process drift or change within the process's design space, actions can be made so that the desired state is always maintained in the NOR area. This is the mission of the Control Strategy part. All data collected during the manufacturing phase are organized and stored in a database which will be used to regularly

reassess risks and improve quality by updating the models used for the predictions. An integrative scheme of the three technological compounds of QbD is presented in Figure 2.

[Figure 2 about here.] 


\subsection{Assessment of the Technological Readiness Level}

The Technological Readiness Level (TRL) is a development scale developed and proposed by the NASA as a systematic metric/measurement system that supports assessments of the maturity of a particular technology and the consistent comparison of maturity between dif-

ferent types of technology. ${ }^{24}$. It was initially used in space technology planning for many years before its use in many other technological fields. Each level corresponds to a specific development milestone. In biomedical engineering, the nine levels are specified in Table 1.

[Table 1 about here.]

\subsection{TRL-indexed Quality-by-Design for Medical Devices}

In this section, we propose a new Quality-by-Design process dedicated to the optimisation (TRL3), maturation (TRL4) and industrialisation (TRL5) of innovative Medical Devices. This new paradigm is presented in Figure 3. The complete risk-based development process is decomposed in nine distinct cycles, noted $\mathrm{QbD} i . j$ where $i \in 1,2,3$ denotes the targeted TRL value and $j \in 1,2,3$ is the risk assessment cycle index within each TRL stage.

[Figure 3 about here.]

- Optimisation Phase.

- Cycle QbD3.1 implements the criticality assessment of risk factors. Results can be described by a Pareto diagram indicating the rank of critical effects.

- Cycle QbD3.2 is an empirical optimisation step. Results are described by a Response Surface Model from which an optimal operating point is determined to minimize or maximize some CQA variables related to efficacy or safety.

- Cycle QbD3.3 performs statistical testing to validate the optimal setting and to potentially establish the proof of concept on a small number of subjects. 
- Maturation Phase. This phase aims at taking into account safety outcomes and at confirming the efficacy of a prototype on a larger number of subjects.

- Cycle QbD4.1 deals with the criticality assessment of risk factors applied to the prototype (TRL3). Results are still described by a Pareto diagram but classification trees and interaction diagrams can also be used to refine the analysis of additive and synergistic effects.

- Cycle QbD4.2 is a risk mapping step. Results are described by a Design Space in which a normal operating region is identified.

- Cycle QbD4.3 performs a validation step of the normal operating region.

- Industrialisation Phase. This phase aims to develop a GMP-manufactured product able to be used in clinical trials.

- Cycle QbD5.1 carries out the scale-up analysis of the design space on the GMPmanufactured product from experimental data collected in GLP context. Those Good Manufacturing Practices and Good Laboratory Practices are mandatory for authorizing clinical trials $(\mathrm{TRL}>5)$.

- Cycle QbD5.2 aims at validating the normal operating space at the industrial level.

- Cycle QbD5.3 implements the process analytical technology (PAT), i.e. the technological compounds to measure the critical process parameters and control their location in the design space. An analytical QbD study is also planned to assess the capability of PAT to provide accurate and reliable measures of CPP. A database management is also included to collect, store, organize and protect manufacturing data. 


\subsection{Realtime Light Controller for Photodynamic Therapy}

Photodynamic therapy (PDT) is an alternative treatment for cancer that involves the administration of a photosensitizing agent, which is activated by light at a specific wavelength ${ }^{25,26,27}$. This illumination causes after a sequence of photoreactions, the production of reactive oxygen species responsible for the death of the tumor cells but also the degradation of the photosensitizing agent, which then loose the fluorescence properties. The phenomenon is commonly known as photobleaching process and can be considered as a therapy efficiency indicator. $\mathrm{In}^{28}$, a real time controller was developed, which enables the tracking of a preset photobleaching trajectory by modulating the light impulses width during the treatment sessions. The biomedical issue of the new medical device is to enable a personalized light dose application during photodynamic therapy but a first proof of concept (TRL3) has to be established and the QbD process presented before has been applied to this study case.

\subsection{Qualitative Crticality Assessment}

To enumerate all potential causes able to affect CQAs, an Ishikawa diagram was firstly produced ${ }^{29}$. This representation consists in grouping causes into major categories to identify and classify these sources of variation.

In a second step, the Failure Modes, Effects and Criticality Analysis was applied to filter and rank the first list of process parameters ${ }^{10}$. FMECA has been around since the 1940s and its role is to identify potential problems that may occur in the manufacturing, assembly and design processes. During this process, the development team sort the risks from highest to lowest through a RPN risk analysis. The RPN (risk priority number) is an estimated metrics of the likelihood of failure. RPN is computed as the product of three quantities: severity (S) of the consequences on the CQA, frequency (F) of occurrence and the degree of non-dectectability (ND) of the cause. 


\subsection{Design of Experiments for QbD3.1: Criticality Assessment}

As illustrated in Figure 3, the QbD3.1 cycle aims at refining the criticality assessment of material attributes and process parameters after the FMECA application. To test the remaining factors, a Plackett-Burman design was chosen to collect informative data with a minimal number of trials and to enable the ranking of their effects on CQAs. In this study case, the six tested factors are described in Figure 4 and their experimental modalities are presented in Table 2. For reasons of simplicity, the structure of the reference trajectory $W(t)$ for the photobleaching trajectory is a straight line. This pattern has the advantage to get only two parameters that are meaningful for the clinician: the targeted photobleaching level $P b_{\text {end }}$ and the treatment duration $T_{D}$. The implemented Plackett-Burman design is described in Table 3. Five replications were used and a randomization of treatment order was applied in each run. Each replicate corresponds to a different position of the optical fiber collecting the fluorescence spectra on the tissue.

[Figure 4 about here.]

[Table 2 about here.]

[Table 3 about here.]

To estimate the additive effects of each factor from the experimental data collected during the Plackett-Burman design, an ANOVA model structure without interaction terms was used:

$$
Y_{l}=b_{0}+\sum_{i=1}^{6} b_{i} u_{i, l}+E_{l},
$$

where $l=1, \cdots, n$ is the number of the experiment in the design, $u_{i}$ is the $i-t h$ factor to be tested, $b_{0}$ is the average response when all factors take their reference level, $b_{i}$ is the effect of the $i$ - th factor while $E_{l} \sim \mathcal{N}\left(0, \sigma^{2}\right)$ is a normally distributed random variable describing the modeling residuals. $Y_{l}$ is a fitting criterion between the reference trajectory, $W\left(t_{k}\right)$, and the observed photobleaching curve, $z\left(t_{k}\right)$ computed over all the discrete time instants $t_{k}$. It 
is computed from the following formula:

$$
Y_{l}=100\left(1-\frac{\left\|W_{l}\left(t_{k}\right)-z_{l}\left(t_{k}\right)\right\|_{2}}{\left\|z_{l}\left(t_{k}\right)-\mu_{z}\right\|_{2}}\right)
$$

with $\mu_{z}$ : the mean value over the replicates of $z_{l}\left(t_{k}\right)$. A perfect tracking of the reference trajectory leads to $Y_{l}=100 \%$.

\subsection{Design of Experiments for QbD3.2: Response Optimization}

After the first risk assessment cycle of ObD3, the second loop presented in Figure 3 only focuses on the previously identified critical parameters. The objective is to identify the values of those impacting factors leading to optimal values of the investigated CQA. To that aim, a quadratic response surface model is used and described as follows in the case of two process parameters:

$$
Y_{l}=b_{0}+\sum_{i=1}^{2} b_{i} u_{i, l}+\sum_{i=1}^{2} b_{i i} u_{i, l}^{2}+b_{12} u_{1, l} u_{2, l}+E_{l}
$$

with: $l=1, \cdots, n$ : is the index of the assay in the design of experiment; $u_{1}=T_{D}$ is the duration of the treatment and $u_{2}=P b_{\text {end }}$ is the targeted endpoint value of the photobleaching trajectory. $n$ : is the total number of experiments to be carried out in the design; $b_{0}$ : value of the mean response; $b_{i}$ : additive effect of the $i$ - th factor; $b_{i i}$ : quadratic effect of the $i-t h$ factor; $b_{12}$ : interaction effect of the two factors on the studied response; $E_{l} \sim \mathcal{N}\left(0, \sigma^{2}\right)$ : modeling residual described by a normally distributed variable.

All the model coefficients have to be estimated from the experimental data collected after experimentation. To that aim, a central composite design was applied to 27 mice. The nine conditions of experimentation of this design are defined in Table 4 and each point was repeated three times.

[Table 4 about here.] 


\subsection{Design of Experiments for QbD3.3: Reproducibility Testing}

The last cycle of the maturation stage is a confirmatory study, which consists in assessing the photobleaching response reproducibility and in comparing it with and without the photobleaching tracking controller. To that aim, we have applied a completely randomized design to compare the reproducibility response between the two groups composed each of 10 subjects. For every run with the controller, different light signals were delivered as shown in Figure 7. The mean cumulated light dose applied to the animals is noted $\mu_{D}\left(\mathrm{~mJ} / \mathrm{cm}^{2}\right)$ and is given by:

$$
\mu_{D}=\operatorname{Irr} \times \frac{\sum_{i=1}^{10} \sum_{t_{k}=0}^{T_{D}} v_{i}\left(t_{k}\right)}{10} \times T_{s}
$$

$I r r=0.16 \mathrm{~mW} / \mathrm{cm}^{2}$ is the light irradiance generated by the LASER, $v_{i}\left(t_{k}\right)$ is the $i-t h$ binary control signal and $T_{s}$ the sampling period. This total light dose was applied 10 times on mice in a continuous illumination pattern (open-loop method). Finally, the reproducibility of each method (with and without controller) was assessed by comparing the variability of $Y$ in the two cases.

\subsection{Animal model}

\subsubsection{Mice}

Experiments were performed on 6 to 12 -weeks-old female nude mice $(\mathrm{nu} / \mathrm{nu})$ weighting between 25 and 30 grams. They were provided by Janvier breeding (Le Genest St Isle, France).

\subsubsection{Photosensitizer}

For this study we used tetraphenyl chlorin (TPC) diluted with a mixture of polyethylene glycol (PEG400), ethanol and water (3:2:5). The injected PS dose was $2 \mathrm{mg} / \mathrm{kg}$ and was performed intravenously. After injection, the mice were kept in dark during a drug-light interval of 24 hours. 


\subsubsection{Experimental protocol}

Experiments were performed under volatile anesthesia consisting in a mixture of isoflurane and air. Induction of the anesthesia is conducted under $4 \%$ of isoflurane and maintained under $2 \%$ during the treatment. During the experiments, mice were placed on a heating plate maintained at $37^{\circ} \mathrm{C}$.

\section{Results}

This section presents the main outcomes of the iQbD process proposed above and applied to the photobleaching controller in photodynamic therapy.

\section{$3.1 \quad$ QTPP}

Intended Application The intended application is the treatment of actinic keratoses, which are thin pre-cancerous lesions that might turn into skin cancer if not treated. These lesions are mainly present on the patient scalp, hands and shoulders.

\section{Device Description}

[Figure 5 about here.]

The closed-loop control scheme of the photobleaching phenomenon during the photodynamic therapy is presented in Figure 5. The objective is to enforce the photobleaching process to follow a reference trajectory. To sum up, this realtime controller shall allow to:

1. reconstruct the photobleaching curve from fluorescence spectra measurements regularly collected during the treatment;

2. automatically adapt the duty cycle of the light impulse signal to track the reference decline trajectory. 
Expected Efficacy the objective is to demonstrate the ability of the photobleaching controller to track a preset reference trajectory.

Quality another aspect to be demonstrated deals with the reproducibility of the photobleaching response.

Contraindication during PDT, pain often manifests as a burning, stinging or prickling sensation and usually peaks in the first minutes of treatment and declines significantly after eight hours ${ }^{30}$.

Preclinical Testing this preclinical study shall to establish the proof of concept of the PDT controller by following up the TRL-indexed QbD approach proposed in section 2.3. In accordance with this new paradigm of development, the maturation stage (QbD3) was applied to the PDT controller.

\subsection{Identification of Critical Process Parameters}

Cause-Effect Modeling The resulting Ishikawa diagram of the qualitative cause-effect modeling of the PDT controller is presented in Figure 6. Some variability factors were gathered to simplify the representation.

[Figure 6 about here.]

Qualitative Criticality Assessment In this step, the different causes enumerated in the cause-effect diagram were analyzed by the FMECA method in which severity, frequency and non-detectability scores were evaluated to compute their risk priority number. The complete FMECA results are given in a supplementary material ${ }^{2}$. The most critical factor is the potential lack of accuracy for the positioning of the optical fiber. Particular attention was brought to fix this background variable during the experimental session. We only kept the

\footnotetext{
${ }^{2}$ https://hal . archives-ouvertes.fr/hal-02937273
} 
factors with a relative criticality index greater to $C=4 \%$. Seven parameters are considered but one of them related to the optical parameters cannot be controlled and its effect is taken into account in the error term of the analysis model defined in (3). The six remaining factors are involved in the empirical criticality analysis presented in the next section.

\subsection{Results of the QbD3.1 cycle}

As illustrated in Figure 3, the QbD3.1 cycle starts by refining the criticality assessment of material attributes and process parameters. After the FMECA application, six risk factors were selected as probably the most critical ones. Table 5 shows results of the ANOVA test applied to experimental data collected during the application of the Plackett-Burman design. One factor, $u_{2}$ (photobleaching trajectory endpoint), appears as to be active ( $b_{2} \approx 145$ with

$\left.p \approx 1.2 \cdot 10^{-9}\right)$. Two other factors could have non-negligible effects: $u_{1}$ : treatment duration and $u_{5}$ : the fluorescence measurement period. The negative estimated value for $b_{5}$ means we have to reduce $u_{5}$ to increase the tracking performance described by $Y$. Accordingly, we fixed $u_{5}$ at its minimum level: $1 s$.

[Table 5 about here.]

\subsection{Results of the QbD3.2 cycle}

A central composite design was applied to the two critical factors previously identified in order to determine their cause-effect relationship with $Y$ whose mean values are given in Table 4.

[Table 6 about here.]

Figure 7 shows the photobleaching trajectories measured during the triplicate application of the central composite design. These data show that the controller is able to support different slopes of the reference trajectory up to a certain point: small $Y$ values (around $30 \%$ ) were observed for fastest (experimental point $(+1,-1))$ and slowest (experimental point $(-\alpha, 0)$ ) 
decreases. Such issues are due to the ON-OFF operating mode of the controller, which does not allow LASER amplitude modulation. Estimation results related to the identification of the response surface model of $Y$ are presented in Table 6. They show that only $u_{2}$ has significant additive $\left(b_{2}\right)$ and quadratic $\left(b_{22}\right)$ effects on $Y$. Finally, the response surface model built from (5) is given in Figure 8 and was used to find out the optimal operating point. The coefficient of determination is equal to $R^{2} \approx 0.62$, which is acceptable to predict optimal settings in in vivo studies.

[Figure 7 about here.]

[Figure 8 about here.]

\subsection{Results of the QbD3.3 cycle}

In order to assess the reproducibility of the photobleaching trajectory tracking with the proposed controller, a confirmatory study were performed with the optimal operating point. At the end of the treatments, the fluorescence index reached a mean of $\mu_{P b_{\text {end }}}=53.3 \%$ $\left(\sigma_{P b_{\text {end }}}=2.3 \%\right)$. The mean total light dose applied for this modality was $\mu_{D}=14.4 \mathrm{~mJ} / \mathrm{cm}^{2}$ $\left(\sigma_{D}=4.7 \mathrm{~mJ} / \mathrm{cm}^{2}\right)$. An equivalent continuous illumination was then performed 10 times in order to obtain the same light dose (approximately 90 seconds).

Figure 9 compares the values of the trajectory tracking performance $(Y)$ in the two control modes: without and with photobleaching trajectory tracking. A statistical summary is presented in Table 7 . All those results clearly emphasize the improved reproducibility of the innovative photobleaching tracking controller in practice and therefore demonstrate the proof of concept.

[Table 7 about here.]

[Figure 9 about here.] 


\section{Discussion}

\subsection{QbD position in relation to FMEA.}

As previously mentioned in introduction, the application of risk management to medical devices is essentially governed by the international standard ISO 14971 in which risk assessment mainly relies on the application of the failure mode and effect analysis. In FMEA, a Risk Priority Number (RPN) is computed by evaluating both the severity and occurence levels of hazards according to predefined scoring scales and declarative information provided by developer teams. A number of FMEA limitations were stressed in the literature ${ }^{11,12,13,14,15}$ but those structural deficiencies do not dismiss it. Moreover, unlike the drug situation, mechanism of action for many medical devices is well understood. The effect tends to be localized rather than systemic and is physical not pharmacokinetic and pharmacodynamic. Additionally, whereas drugs are discovered and basically don't change at all, devices evolve and are constantly changing. Therefore, FMEA is better suited to be applied to medical devices than drugs. Nevertheless, if FMEA can help to identify which parameters are more or less critical, there is a need to complete it by a complementary approach able to refine the estimation of critical risks through the application of data-driven modeling techniques. In this study, Quality-by-Design was assessed as a potential candidate to carry out this mission.

In the Quality by Design framework, the risk assessment relies on the estimation of probabilities to fulfill specifications related to efficacy, safety and quality requirements. Those probabilities requires the measurement and modeling of critical quality attributes from data collected during experimental studies with statistically designed protocols. The design of experiments can be applied from the first investigation tests to provide unbiased results from a small amount of assays. This is why QbD can be defined as a data-driven and model-based approach of risk assessment in the context of product development. The use of experimental data and empirical models is a key difference from FMEA.

Another facet of the complementarity between the two approaches concerns the risk mitigation, since the QbD cycle includes a step entitled Process Analytical Technology used 
in conjunction with a process control strategy to adapt in line values of some critical process parameters to maintain the operating point within a desired region of the design space.

\subsection{QbD adaptation for medical device engineering.}

Unlike pharmaceuticals, very few applications of QbD exist for medical devices. One major issue is to adapt the risk-based engineering to the development needs, which differ according to the technological readiness level: TRL3 consists in establishing a proof of concept while TRL5 aims at validating a GMP-manufactured product tested in GLP framework. The key contribution of this study is to propose a double hierarchical decomposition of the conventional QbD workflow to better select the most appropriate risk assessment methods and tools at each development step. The new development paradigm is entitled TRL-indexed QbD (iQbD) and is split up into three main stages associated each with TRL: 3, 4 and 5. Within each stage, three consecutive data-driven risk assessment cycles have been proposed to efficiently address the question raised. For the TRL3 stage, goals of the three consecutive cycles are to perform criticality assessment, efficacy optimisation and validation to establish an empirical proof of concept. For the TRL5 stage, the three cycles aim at identifying the design space at industrial scale, validating it and implementing technology for the control strategy (risk mitigation). Each risk assessment cycles involves the implementation of experimental designs and multivariate regression/classification techniques. Each cycle can be replicated several times before validating the results and moving on to the next one. The iQbD organization in two decomposition levels also clarifies the development workflow and its successive outcomes. This better readability should contribute to increase its applicability to the development of innovative medical devices.

In the previous section, we have applied the proposed iQbD paradigm to the development of a new medical device: a realtime photobleaching controller developed to improve the reproducibility of the therapeutic response in photodynamic therapy. More precisely, the objective was to demonstrate the proof of concept in laboratory of the innovative controller through in vivo tests. Accordingly, only the QbD3 stage was implemented and its three 
learning cycles were executed to identify critical factors, determine optimal settings, validate them and finally establish the proof of concept. This real application has shown how this iterative and risk-based development method allowed us to reach TRL3 in only three risk assessment cycles, which emphasizes its practical relevance. However for simple medical devices or components, the application of the three consecutive empirical investigation cycles is not mandatory and only one can be enough, e.g. when the number risk factors is small.

In the near future, the objective of the subsequent QbD4 study will be to demonstrate the efficacy of the closed-loop PDT strategy and to examine how it could reduce the pain due to treatment. Other applications to medical devices under development at TRL4 and 5 are on going in the TBMED project ${ }^{3}$. Although the scope of the iQbD approach initially covers the whole preclinical phase, convergences in the clinical context have been recently proposed $^{31,32,33}$ and provide promising perspectives to extend iQbD up to TRL 8. One important perspective of investigation consists in determining more accurately how QbD fits in the FDA Design Controls (21.CFR.820.30) for Medical Devices. In particular, a roadmap is still needed to use the $\mathrm{QbD}$ and FDA design control requirements for the risk-based development of drug-device combination products.

\subsection{QbD application scope.}

Engineering risks involved in pharmaceutical and medical device development can be handled by QbD. ICH Q8 ${ }^{34}$ and Q11 ${ }^{35}$ guidelines clearly split up two categories of input risk factors considered in QbD: the critical material attributes essentially associated with the product design and the critical process parameters related to the manufacturing step. Since a few years, the international committee of harmonization has also introduced the quality by design in its guideline for good clinical practice: ICH E6 ${ }^{36}$. However, human activities and usability in particular but also management and business risks are not covered by QbD.

\footnotetext{
${ }^{3}$ https://www.tbmed.eu/
} 


\subsection{QbD economical benefits.}

Another line of investigation is to assess the return on investment of $\mathrm{QbD}$ analysis. Its purpose is not to speed up the engineering process but to avoid bad estimation of risks that could postpone the product development and so cause critical financial consequences for the developer. However, in practice, since 2012 and a survey carried out on 12 companies $^{37}$, no other economical study was performed. As mentioned in ${ }^{38}$, during the early stages of QbD introduction, the business case was based on qualitative potential benefits in a few key areas: minimized manufacturing-scale development studies, fewer quality issues, greater flexibility to optimize postlicensure, improved patient-focus for the product, more clinically meaningful product specifications, and reduced effort in regulatory interactions. Nevertheless, the business case for QbD can be difficult to establish because QbD has several tangible and intangible benefits that are challenging to quantify. This pending issue not only concerns pharmaceutical products but also medical devices.

\section{Conflict of Interest}

I hereby disclose all of my conflicts of interest and other potentially conflicting interests. With regard to my position as a co-founder of CYBERnano, a start-up founded in 2013 and specialized in Quality-by-Design, my mission has been to transfer the scientific knowhow in this field from the Université de Lorraine to the young company in compliance with a contract signed up by the two parties. This contract notably specifies the prohibition to have a leading position in this company. I have no employment in this corporation, I have not provided any consultancy or have not collected fees from it since its creation. I hereby confirm that the disclosure made above are complete and correct to the best of my information and belief. 


\section{Acknowledgment}

This project has received funding from the European Union's Horizon 2020 research and innovation programme under grant agreement no. 814439. The author is solely responsible for its content, it does not represent the opinion of the European Commission and the Commission is not responsible for any use that might be made of data appearing therein.

\section{References}

1. Medina, L. A., Kremer, G. E. O., and Wysk, R. A., 2013. "Supporting medical device development: a standard product design process model". Journal of Engineering Design, 24(2), pp. 83-119.

2. Ham, S. T., 2010. "Mapping the Medical Device Development Process".

3. Santos, I. C., Gazelle, G. S., Rocha, L. A., and Tavares, J. M. R., 2012. "An ontology model for the medical device development process in europe". In The 1st International Conference on Design and PROcesses for MEdical Devices-PROMED 2012.

4. Buzan, T., 2013. Mind map handbook: The ultimate thinking tool. HarperCollins UK.

5. Mollberg, N., Surati, M., Demchuk, C., Fathi, R., Salama, A., Husain, A., Hensing, T., and Salgia, R., 2011. "Mind-mapping for lung cancer: towards a personalized therapeutics approach". Advances in therapy, 28(3), pp. 173-194.

6. Juran, J. M., 1993. Quality planning and analysis; from product development through use. No. 04; TS156, J8 1993.

7. Elahi, B., 2018. Safety Risk Management for Medical Devices. Academic Press.

8. Singh, K., and Selvam, P., 2020. "Medical device risk management". In Trends in Development of Medical Devices. Elsevier, pp. 65-76. 
9. Committee for Human Medicinal Products, 2015. ICH guideline Q9 on quality risk management. Tech. Rep. Step 5, European Medicine Agency, September.

10. Stamatis, D. H., 2003. Failure mode and effect analysis: FMEA from theory to execution. Quality Press.

11. Liu, H.-C., Liu, L., and Liu, N., 2013. "Risk evaluation approaches in failure mode and effects analysis: A literature review". Expert systems with applications, 40(2), pp. 828838.

12. Driessen, M., Arts, J., van Houtum, G.-J., Rustenburg, J. W., and Huisman, B., 2015. "Maintenance spare parts planning and control: a framework for control and agenda for future research". Production Planning \& Control, 26(5), pp. 407-426.

13. Mentes, A., and Ozen, E., 2015. "A hybrid risk analysis method for a yacht fuel system safety". Safety science, 79, pp. 94-104.

14. Brun, A., and Savino, M. M., 2018. "Assessing risk through composite fmea with pairwise matrix and markov chains". International Journal of Quality 86 Reliability Management.

15. Subriadi, A. P., and Najwa, N. F., 2020. "The consistency analysis of failure mode and effect analysis (fmea) in information technology risk assessment". Heliyon, 6(1), p. e03161.

16. Powell, T. C., 1995. "Total quality management as competitive advantage: a review and empirical study". Strategic management journal, 16(1), pp. 15-37.

17. Bergman, B., and Klefsjo, B., 2010. Quality from customer needs to customer satisfaction. Studentlitteratur AB.

18. Yang, K., and El-Haik, B., 2003. Design for Six Sigma. McGraw-Hill New York.

19. Juran, J., 1992. Juran on Quality by Design: The New Steps for Planning Quality into Goods and Services. Free Press. 
20. ICH Expert Working Group, 2009. Pharmaceutical Development Q8(R2). International Conference on Harmonisation, August.

21. Food, U., Administration, D., et al., 1997. "Design control guidance for medical device manufacturers". Center for Devices and Radiological Health.

22. Rivero, A. M., Monteiro, E. C., Leite, D. S., Tannenbaum, F. S., Pinto, M. A., and Leite, K., 2015. "Implementing first stages of quality by design approach in the development of an assistive robotics technology". In XXI IMEKO World Congress, pp. 1295-1299.

23. Martinez-Marquez, D., Mirnajafizadeh, A., Carty, C. P., and Stewart, R. A., 2018. "Application of quality by design for $3 \mathrm{~d}$ printed bone prostheses and scaffolds". PloS one, 13(4), p. e0195291.

24. Mankins, J. C., 1995. "Technology readiness levels". White Paper, April, 6, p. 1995.

25. Moser, J. G., 1998. Photodynamic Tumor Therapy: 2nd and 3rd Generation. Gordon \& Breach Science Publishers.

26. Dougherty, T., Gomer, C. J., Henderson, B. W., Jori, G., Kessel, D., Korbelik, M., Moan, J., and Peng, Q., 1998. "Photodynamic therapy". Journal of the National Cancer Institute, 90(12), June, pp. 889-905.

27. Goldman, M. P., 2005. Photodynamic Therapy. W.B. Saunders Company.

28. Tylcz, J.-B., Bastogne, T. T., Bourguignon, A., Frochot, C., and Barberi-Heyob, M., 2016. "Realtime tracking of the Photobleaching Trajectory during Photodynamic Therapy". IEEE Transactions on Biomedical Engineering.

29. Wong, K. C., 2011. Using an ishikawa diagram as a tool to assist memory and retrieval of relevant medical cases from the medical literature.

30. Fink, C., Enk, A., and Gholam, P., 2015. "Photodynamic therapy-aspects of pain management". JDDG: Journal der Deutschen Dermatologischen Gesellschaft, 13(1), pp. $15-22$. 
31. Agency, E. M., 2013. "Reflection paper on risk based quality management in clinical trials". Compliance Insp, 44, pp. 1-15.

32. Hurley, C., Shiely, F., Power, J., Clarke, M., Eustace, J. A., Flanagan, E., and Kearney, P. M., 2016. "Risk based monitoring (rbm) tools for clinical trials: a systematic review". Contemporary clinical trials, 51, pp. 15-27.

33. Huang, G. D., Bull, J., McKee, K. J., Mahon, E., Harper, B., Roberts, J. N., et al., 2018. "Clinical trials recruitment planning: a proposed framework from the clinical trials transformation initiative". Contemporary clinical trials, 66, pp. 74-79.

34. Committee for Human Medicinal Products, 2017. ICH guideline Q8 (R2) on pharmaceutical development. Tech. Rep. Step 5, European Medicine Agency, June 22.

35. Committee for Human Medicinal Products, 2012. ICH guideline Q11 on development and manufacture of drug substances (chemical entities and biotechnological/biological entities). Tech. Rep. Step 5, European Medicine Agency, November.

36. Committee for Human Medicinal Products, 2016. Guideline for good clinical practice E6(R2). Tech. Rep. Step 5, European Medicine Agency, December 1.

37. Kourti, T., and Davis, B., 2012. "The business benefits of quality by design (qbd)". Pharmaceutical Engineering, 32, 07, pp. 52-62.

38. Junker, B. H., 2012. Building a business case for biopharmaceutical qbd implementation (peer reviewed). BioPharm International Magazine, http://www.processdevelopmentforum.com/articles/building-a-business-case-forbiopharmaceutical-qbd-implementation-peer-reviewed/, August.

39. Morales, J. M. H., 2015. "Evaluating biocompatible barrier films as encapsulants of medical micro devices". PhD thesis, Université Grenoble Alpes. 


\section{List of Figures}

1 Design Space Stability . . . . . . . . . . . . . . . . . . . . . . 28

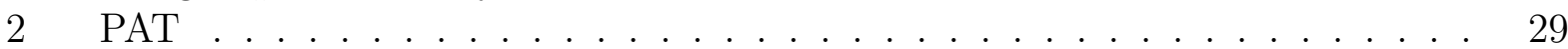

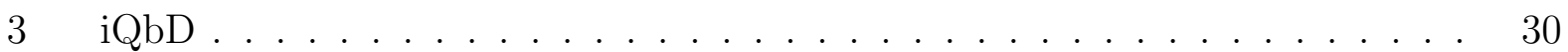

4 Set of critical process parameters to be tested. . . . . . . . . . . . . . 31

5 A closed-loop control scheme of the photobleaching control in PDT. The photobleaching controller compares the measured photobleaching trajectory (green) with the reference curve (red) and modulates the width of light impulses $L(t)$ to minimize the tracking error. $P S(t)$ is a step signal describing the administration of a preset amount of photosensitizing drug at time instant: $t=0 . O_{2}(t)$ is the amount of oxygen is the illuminated tissue and is considered as a disturbance variable. $S(t)$ is the output variable and corresponds to the viability of cells at time $t \ldots \ldots \ldots \ldots \ldots$

6 Cause-effect diagram of the Photobleaching Controller on the Tracking Performance . . . . . . . . . . . . . . . . . . . . .

$7 \quad$ Nine sets of photobleaching responses measured during the central composite design. Each experimental condition was repeated three times. . . . . . . . .

8 Response surface of $Y(\%)$ deduced from response predictions computed from model $(5) . u_{1}=T_{D}(\min )$ and $u_{2}=P b_{\text {end }}(\%) \ldots \ldots \ldots$

9 Box-plots of $Y$ characterizing the reproducibility of the photobleaching responses for the common PDT treatment and with the photobleaching controller. They show a significant reduction of the output variability $(p<0.01)$ when the photobleaching tracking controller is used. . . . . . . . . . 


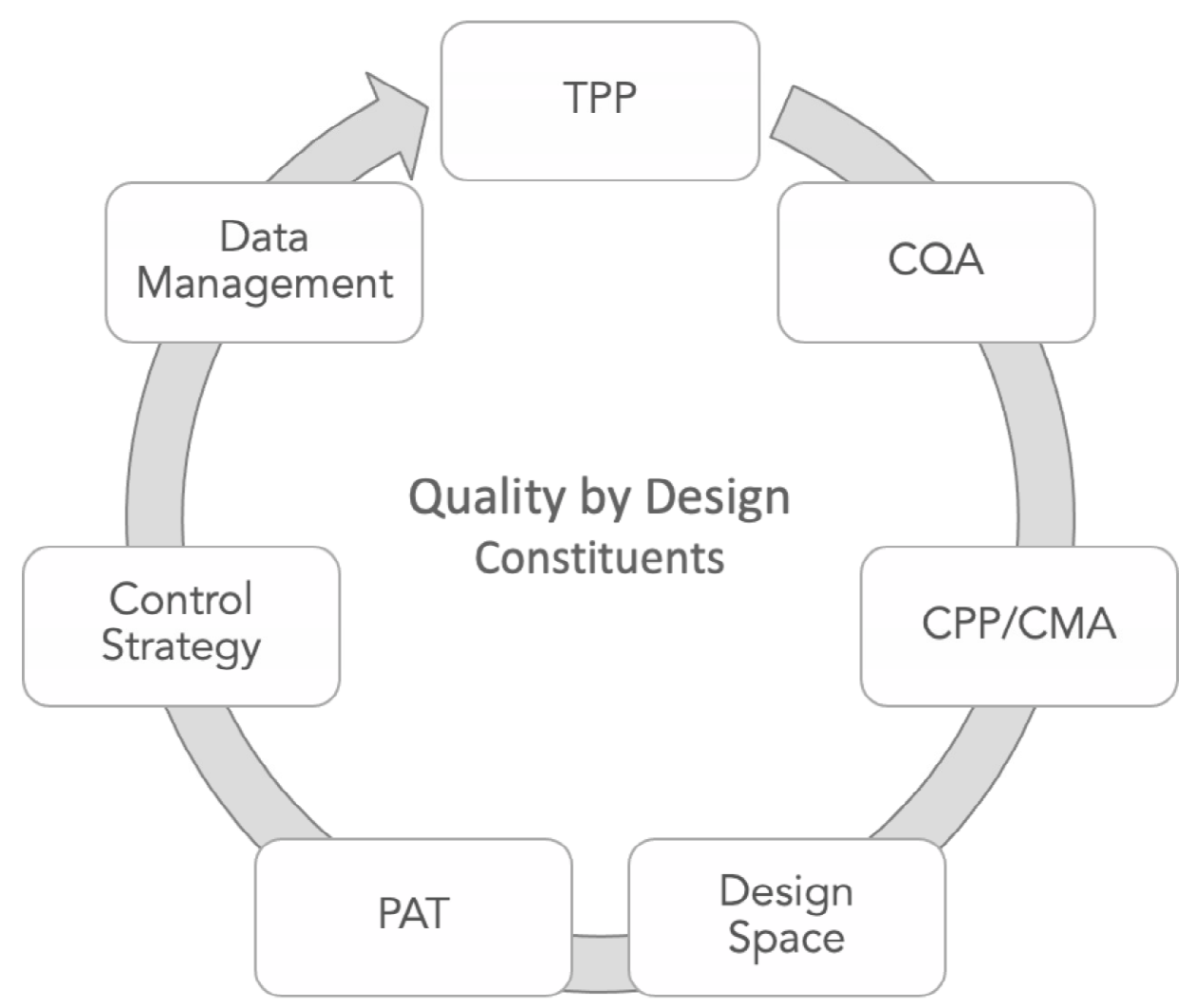

Figure 1: Quality-by-Design constituents: QTPP (Quality Target Product Profile), CQA (Critical Quality Attributes), CMA (Critical Material Attributes), CPP (Critical Process Parameters), Design Space, PAT (Process Analytical Technology), Control Strategy, and Data Management for Continuous Improvement. 


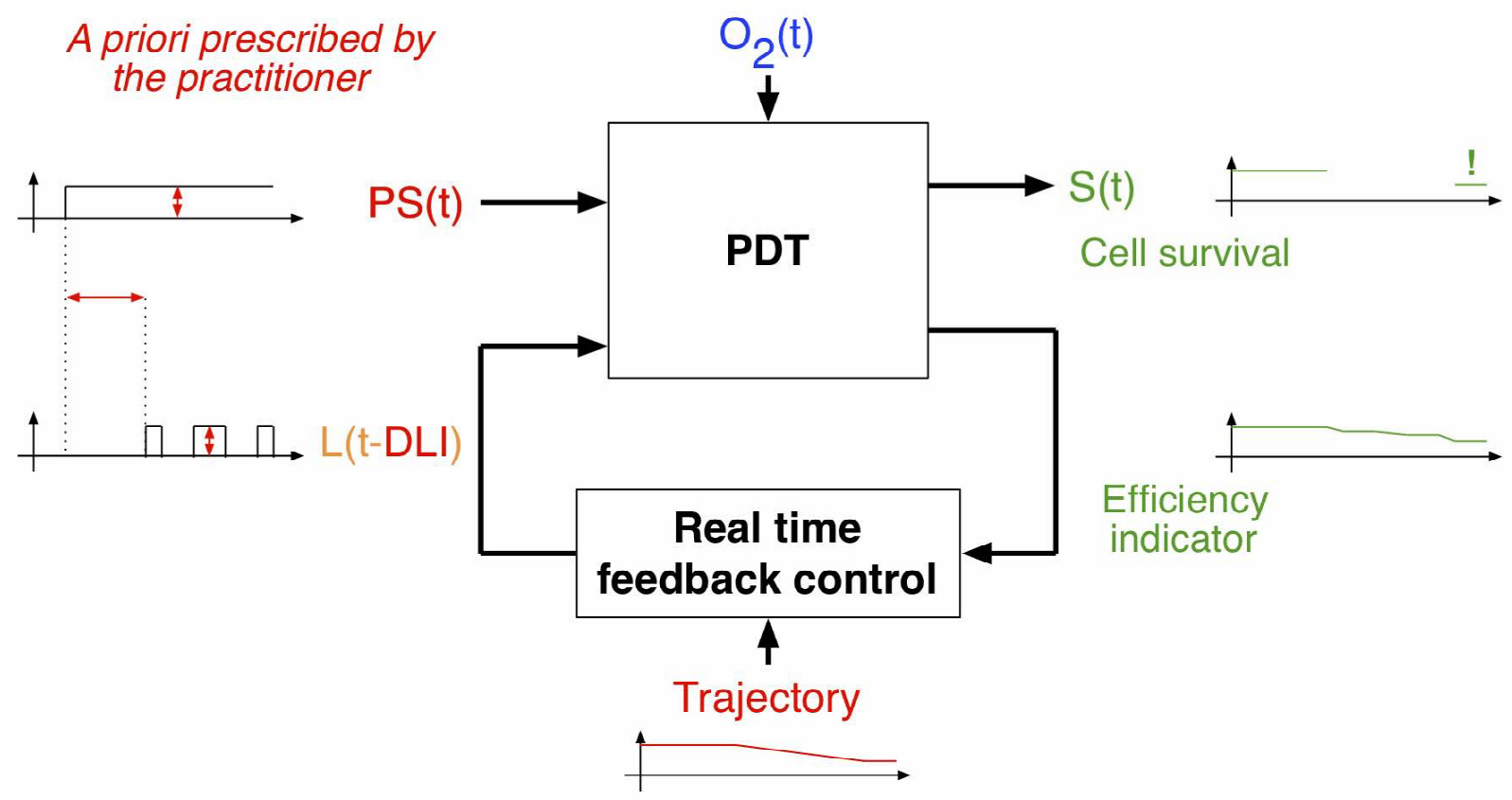

Figure 2: Organization of the technological compounds in QbD to implement the measurement, the storage and the control of critical process parameters. 


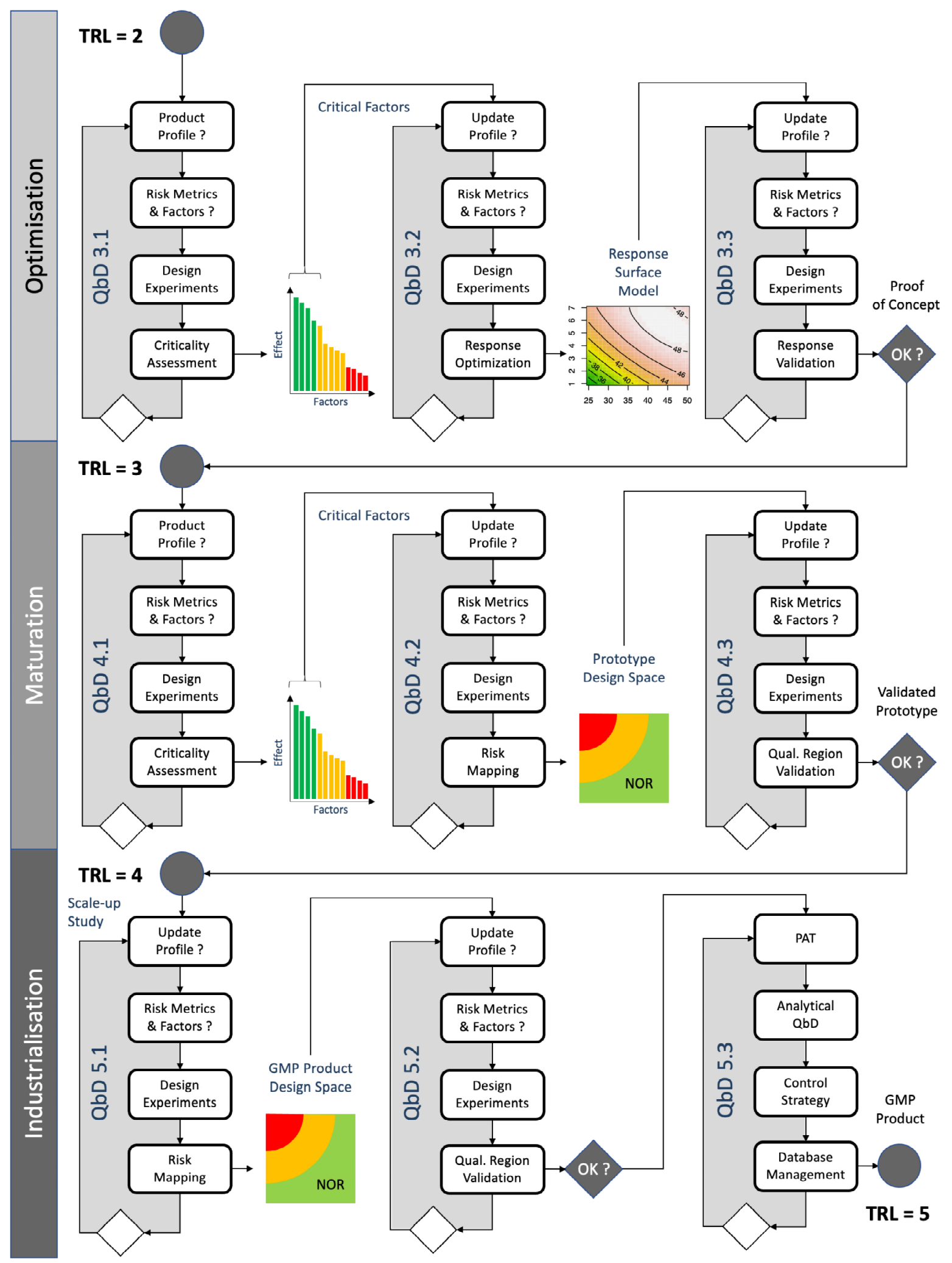

Figure 3: QbD Implementation Process indexed according to the Technological Readiness Level of the product to be developed. iQbD is decomposed into three stages: optimisation (TRL3), maturation (TRL4) and industrialisation (TRL5). Each TRL stage is also decomposed into three consecutive data-driven risk assessment cycles. Each cycle can be performed several times according to the results of the validation tests. 


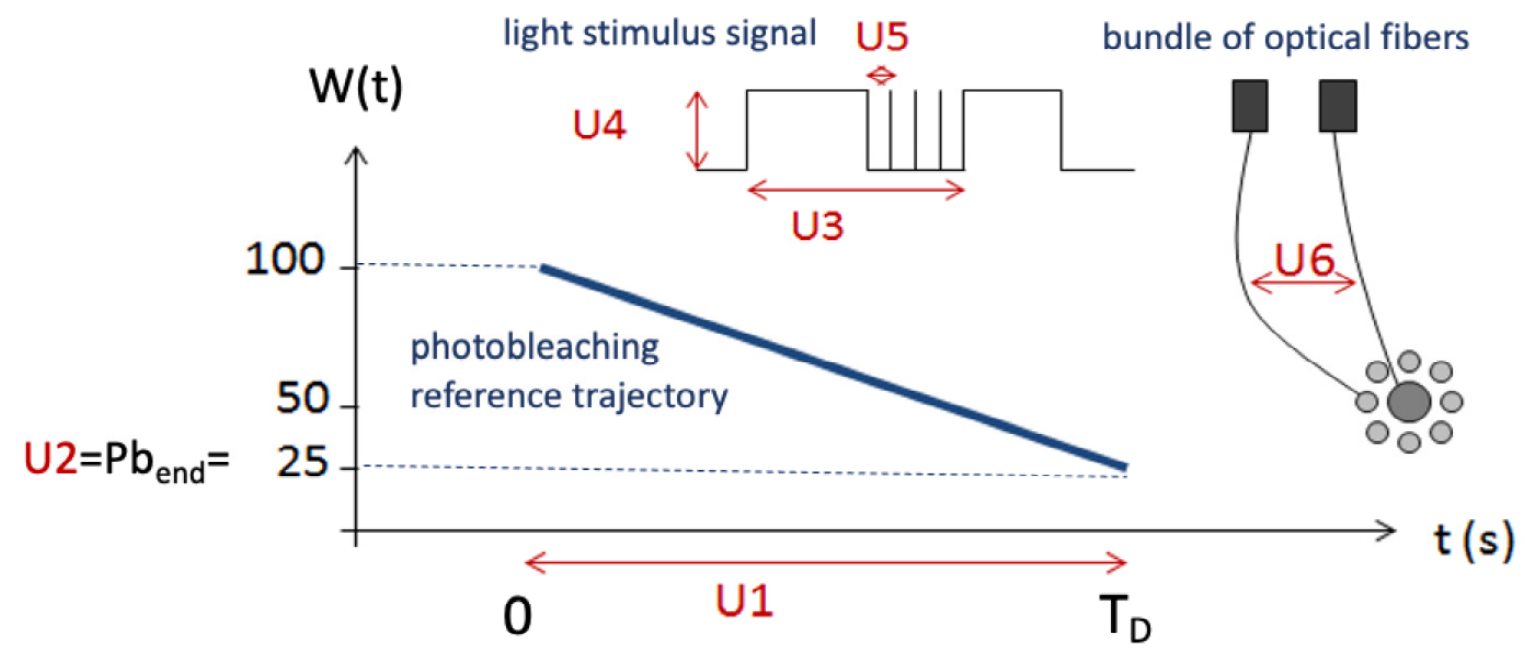

Figure 4: Set of critical process parameters to be tested. 


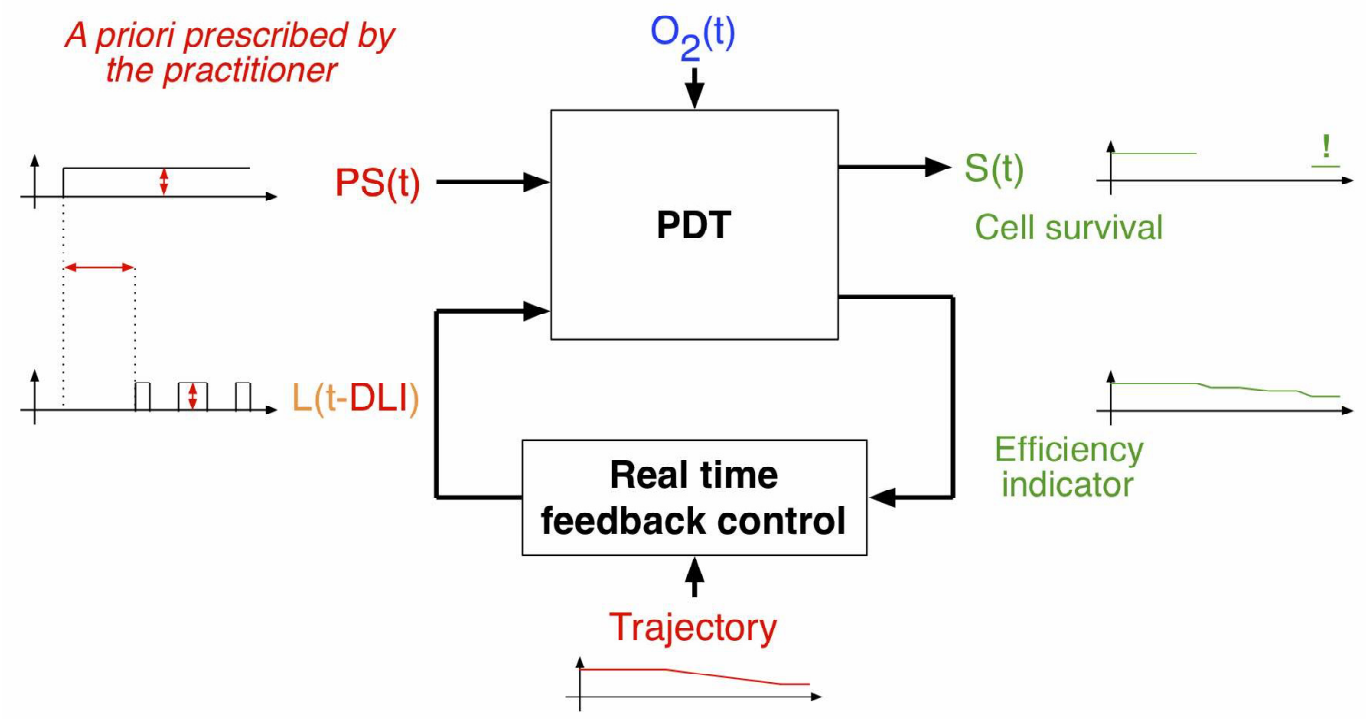

Figure 5: A closed-loop control scheme of the photobleaching control in PDT. The photobleaching controller compares the measured photobleaching trajectory (green) with the reference curve (red) and modulates the width of light impulses $L(t)$ to minimize the tracking error. $P S(t)$ is a step signal describing the administration of a preset amount of photosensitizing drug at time instant: $t=0 . \mathrm{O}_{2}(t)$ is the amount of oxygen is the illuminated tissue and is considered as a disturbance variable. $S(t)$ is the output variable and corresponds to the viability of cells at time $t$. 

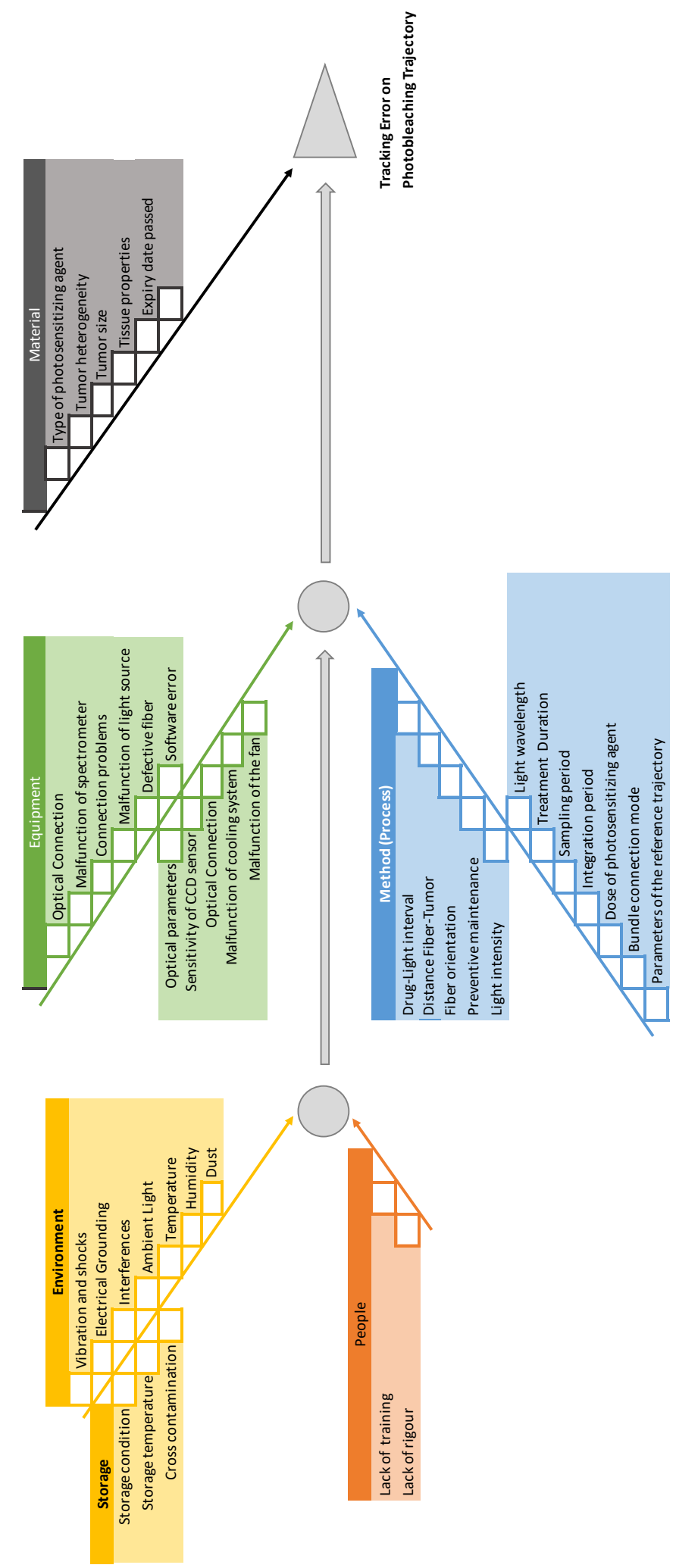

Figure 6: Cause-effect diagram of the Photobleaching Controller on the Tracking Performance 

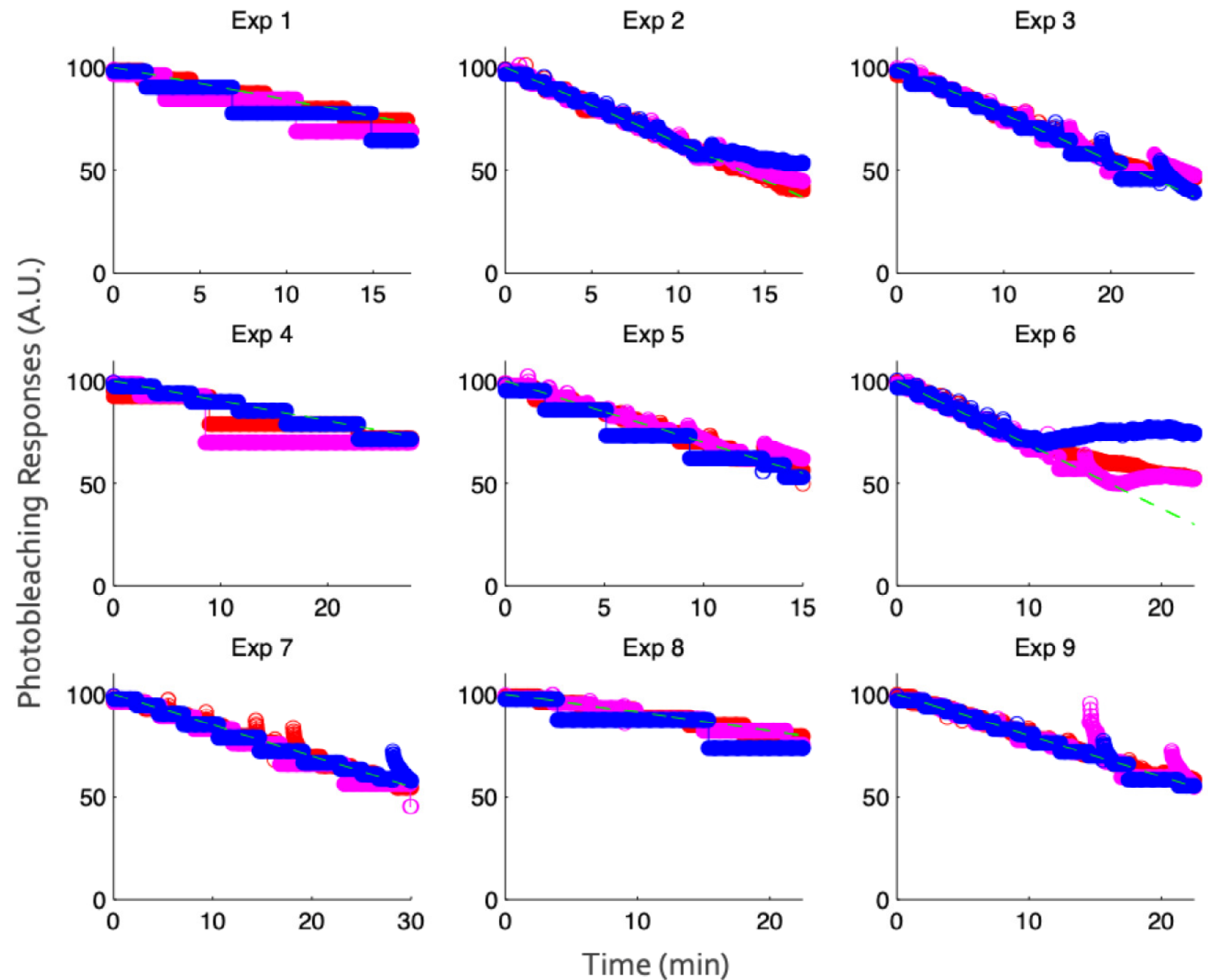

Figure 7: Nine sets of photobleaching responses measured during the central composite design. Each experimental condition was repeated three times. 


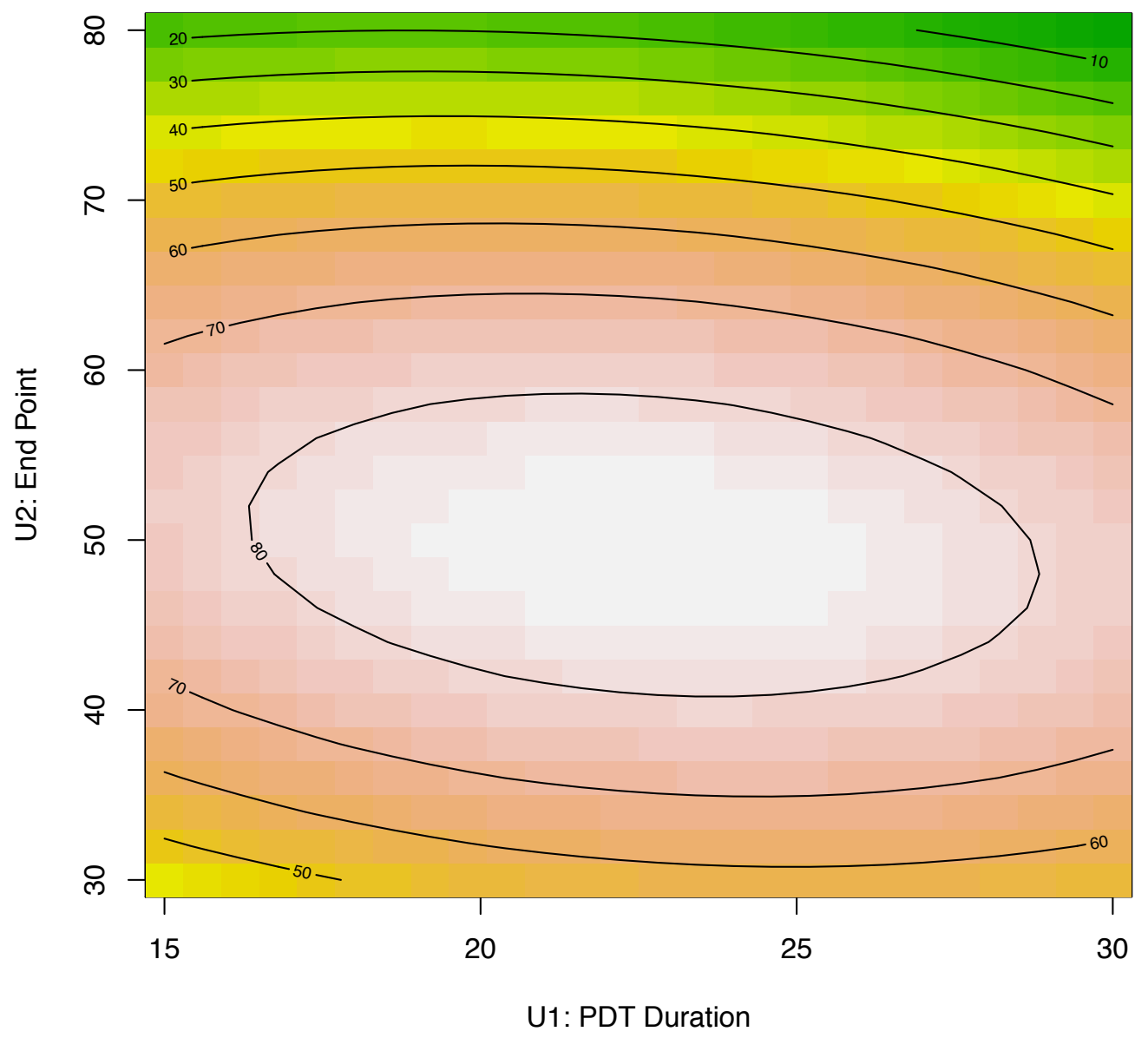

Figure 8: Response surface of $Y(\%)$ deduced from response predictions computed from model (5). $u_{1}=T_{D}(\min )$ and $u_{2}=P b_{\text {end }}(\%)$ 


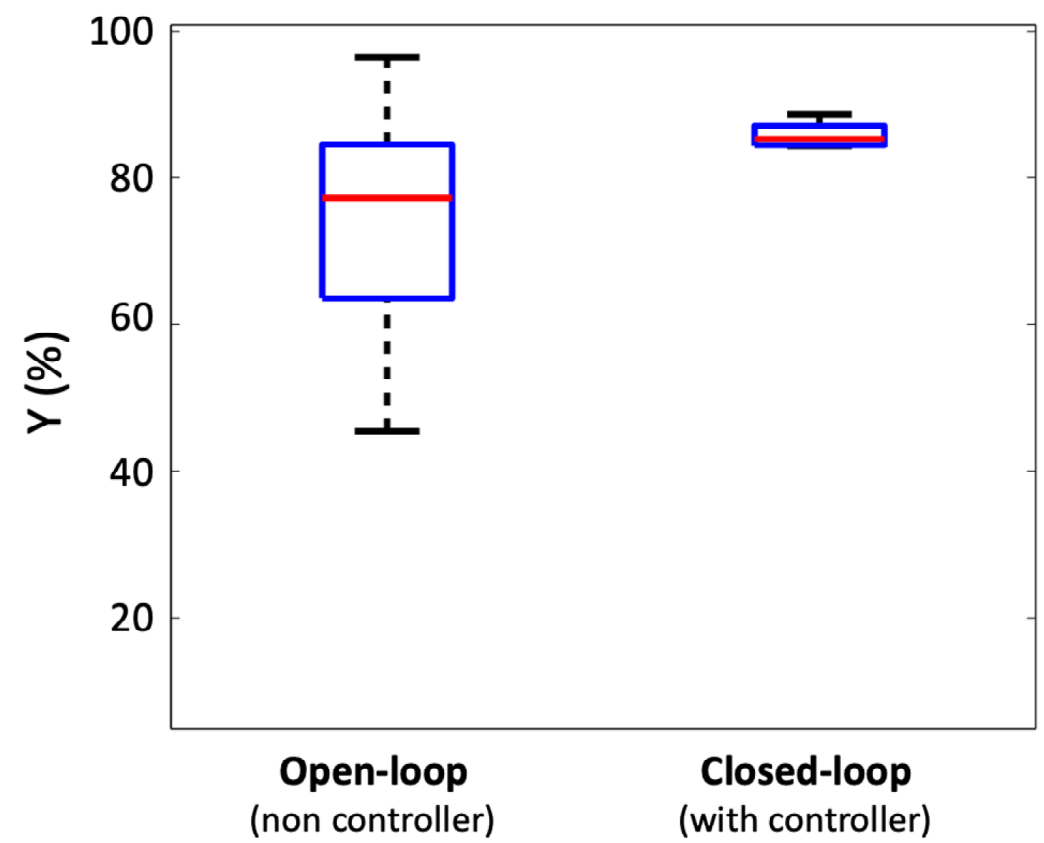

Figure 9: Box-plots of $Y$ characterizing the reproducibility of the photobleaching responses for the common PDT treatment and with the photobleaching controller. They show a significant reduction of the output variability $(p<0.01)$ when the photobleaching tracking controller is used. 


\section{List of Tables}

1 TRL scale for the development of innovative medical devices and drugs ${ }^{39}$. MD: Medical Device. GMP: Good Manufacturing Practice. GLP: Good Laboratory Practice. . . . . . . . . . . . . . . . . . . . . . 38

2 Description of factors involved in the design of experiments devoted to the criticality assessment . . . . . . . . . . . . . . . . . 39

3 Description of the Design of Experiments based on a Plackett-Burman (Hadamard) matrix. The design was replicated five times in a randomized order of the experimental conditions. Each replicate corresponds to a different position of the optical fiber collecting the fluorescence spectra on the tissue. . . . . . . . 40

4 Experimental points coordinates and the corresponding mean fitting coefficients $(n=3) \ldots \ldots \ldots \ldots$. . . . . . . . . . . . . . . . . . . . . . . . . . . . . .

5 Results of the ANOVA analysis. . . . . . . . . . . . . . . . . . . . 42

$6 \quad$ Results of the parameter estimation applied to the response surface model. . 43

7 Main statistics (average and standard deviation) of the fitting coefficients $Y$ for open- (without controller) and closed-loop (with controller) modes. . . . 44 


\begin{tabular}{l|l}
\hline TRL & Technological Milestone \\
\hline \hline 1 & Basic principles observed and reported \\
\hline 2 & Technology concept and/or application formulated \\
\hline 3 & $\begin{array}{l}\text { Initial Proof-of-Concept (established on a small number } \\
\text { of observations) }\end{array}$ \\
\hline 4 & $\begin{array}{l}\text { Validated prototype whose efficacy and safety have been } \\
\text { validated in laboratory on a large number of experimen- } \\
\text { tal units. }\end{array}$ \\
\hline 5 & $\begin{array}{l}\text { GMP product whose efficacy and safety were tested in } \\
\text { GLP context. }\end{array}$ \\
\hline 6 & Feasibility study (MD) / Phase I study (Drug) \\
\hline 7 & Pivotal study (MD) / Phase II study (Drug) \\
\hline 8 & Pivotal study (MD) / Phase III study (Drug) \\
\hline 9 & Access to Market \\
\hline
\end{tabular}

Table 1: TRL scale for the development of innovative medical devices and drugs ${ }^{39}$. MD: Medical Device. GMP: Good Manufacturing Practice. GLP: Good Laboratory Practice. 


\begin{tabular}{|c|c|c|c|c|}
\hline & Factor & $\mathrm{Nb}$ of levels & Levels & Value \\
\hline \multirow{2}{*}{ U1 } & \multirow{2}{*}{ PDT Duration } & \multirow{2}{*}{2} & 1 & $900 \mathrm{~s}$ \\
\hline & & & 2 & $1800 \mathrm{~s}$ \\
\hline \multirow{2}{*}{ U2 } & \multirow{2}{*}{ Trajectory endpoint } & \multirow{2}{*}{2} & 1 & $25 \%$ \\
\hline & & & 2 & $50 \%$ \\
\hline \multirow{2}{*}{ U3 } & \multirow{2}{*}{ Signal Period } & \multirow{2}{*}{2} & 1 & $30 \mathrm{~s}$ \\
\hline & & & 2 & $60 s$ \\
\hline \multirow{2}{*}{ U4 } & \multirow{2}{*}{ Intensity } & \multirow{2}{*}{2} & 1 & $10 \mathrm{~A}$ \\
\hline & & & 2 & $15 \mathrm{~A}$ \\
\hline \multirow{2}{*}{ U5 } & \multirow{2}{*}{ Measurement Period } & \multirow{2}{*}{2} & 1 & $1 \mathrm{~s}$ \\
\hline & & & 2 & $3 \mathrm{~s}$ \\
\hline \multirow{2}{*}{ U6 } & \multirow{2}{*}{ Bundle Connection } & \multirow{2}{*}{2} & 1 & Spectro internal \\
\hline & & & 2 & Spectro external \\
\hline
\end{tabular}

Table 2: Description of factors involved in the design of experiments devoted to the criticality assessment 


\begin{tabular}{|c|c|c|c|c|c|c|c|c|}
\hline \multicolumn{8}{|c|}{ Plackett-Burman Design - Replicate 1} & \multirow{3}{*}{$\mathbf{Y}$} \\
\hline$N^{\circ} \operatorname{Exp}$ & Rand & U1 & U2 & U3 & U4 & U5 & U6 & \\
\hline & & $\mathrm{s}$ & $\%$ & $\mathrm{~s}$ & $\mathrm{~A}$ & $\mathrm{~s}$ & Spectro. & \\
\hline 4 & 1 & 1800 & 25 & 30 & 15 & 3 & Ext. & 5984.80 \\
\hline 5 & 2 & 900 & 50 & 30 & 10 & 3 & Ext. & 4965.60 \\
\hline 2 & 3 & 900 & 50 & 60 & 15 & 1 & Ext. & 3536.30 \\
\hline 6 & 4 & 1800 & 25 & 60 & 10 & 1 & Ext. & 4310.30 \\
\hline 3 & 5 & 900 & 25 & 60 & 15 & 3 & Int. & 4801.20 \\
\hline 8 & 6 & 900 & 25 & 30 & 10 & 1 & Int. & 1867.20 \\
\hline 7 & 7 & 1800 & 50 & 30 & 15 & 1 & Int. & 2186.80 \\
\hline 1 & 8 & \begin{tabular}{|l|}
1800 \\
\end{tabular} & 50 & 60 & 10 & 3 & Int. & 4538.20 \\
\hline \multicolumn{8}{|c|}{ Plackett-Burman Design - Replicate 2} & \multirow{3}{*}{$\mathbf{Y}$} \\
\hline$N^{\circ} \operatorname{Exp}$ & Rand & U1 & U2 & U3 & U4 & U5 & U6 & \\
\hline & & $\mathrm{s}$ & $\%$ & $\mathrm{~s}$ & $\mathrm{~A}$ & $\mathrm{~s}$ & Spectro. & \\
\hline 5 & 1 & 900 & 50 & 30 & 10 & 3 & Ext. & 3631.4 \\
\hline 1 & 2 & 1800 & 50 & 60 & 10 & 3 & Int. & 4436.7 \\
\hline 7 & 3 & 1800 & 50 & 30 & 15 & 1 & Int. & 4130.4 \\
\hline 4 & 4 & 1800 & 25 & 30 & 15 & 3 & Ext. & 5560.3 \\
\hline 3 & 5 & 900 & 25 & 60 & 15 & 3 & Int. & 5645.6 \\
\hline 2 & 6 & 900 & 50 & 60 & 15 & 1 & Ext. & 4883.4 \\
\hline 6 & 7 & 1800 & 25 & 60 & 10 & 1 & Ext. & 5796.3 \\
\hline 8 & 8 & \begin{tabular}{|l}
900 \\
\end{tabular} & 25 & 30 & 10 & 1 & Int. & 7622.9 \\
\hline \multicolumn{8}{|c|}{ Plackett-Burman Design - Replicate 3} & \multirow{3}{*}{$\mathbf{Y}$} \\
\hline $\mathbf{N}^{\circ} \operatorname{Exp}$ & Rand & Duree_PDT & $\begin{array}{c}\text { End_Point_P } \\
\text { DT }\end{array}$ & $\begin{array}{l}\text { Periode_ } \\
\text { Signal }\end{array}$ & Intensité & $\begin{array}{l}\text { Periode_ } \\
\text { Inte }\end{array}$ & Bundle & \\
\hline & & $\mathrm{s}$ & \begin{tabular}{|c|}
$\%$ \\
\end{tabular} & $\mathrm{~s}$ & $\mathrm{~A}$ & $\mathrm{~s}$ & Spectro. & \\
\hline 1 & 1 & 1800 & 50 & 60 & 10 & 3 & Int. & 2798.8 \\
\hline 4 & 2 & 1800 & 25 & 30 & 15 & 3 & Ext. & 6206.3 \\
\hline 2 & 3 & 900 & 50 & 60 & 15 & 1 & Ext. & 2407.5 \\
\hline 3 & 4 & 900 & 25 & 60 & 15 & 3 & Int. & 4548.1 \\
\hline 8 & 5 & 900 & 25 & 30 & 10 & 1 & Int. & 4818 \\
\hline 5 & 6 & 900 & 50 & 30 & 10 & 3 & Ext. & 3208.2 \\
\hline 7 & 7 & 1800 & 50 & 30 & 15 & 1 & Int. & 3374.4 \\
\hline 6 & 8 & 1800 & 25 & 60 & 10 & 1 & Ext. & 3219.3 \\
\hline \multicolumn{8}{|c|}{ Plackett-Burman Design - Replicate 4} & \multirow{3}{*}{ Y } \\
\hline$N^{\circ} \operatorname{Exp}$ & Rand & U1 & U2 & U3 & U4 & U5 & U6 & \\
\hline & & $s$ & $\%$ & $\mathrm{~s}$ & $\mathrm{~A}$ & $\mathrm{~s}$ & Spectro. & \\
\hline 2 & 1 & 900 & 50 & 60 & 15 & 1 & Ext. & 3187.9 \\
\hline 3 & 2 & 900 & 25 & 60 & 15 & 3 & Int. & 5164.8 \\
\hline 6 & 3 & 1800 & 25 & 60 & 10 & 1 & Ext. & 7164.2 \\
\hline 1 & 4 & 1800 & 50 & 60 & 10 & 3 & Int. & 4603.7 \\
\hline 5 & 5 & 900 & 50 & 30 & 10 & 3 & Ext. & 9923.5 \\
\hline 8 & 6 & 900 & 25 & 30 & 10 & 1 & Int. & 4762.1 \\
\hline 4 & 7 & 1800 & 25 & 30 & 15 & 3 & Ext. & 5279.1 \\
\hline 7 & 8 & \begin{tabular}{|l|}
1800 \\
\end{tabular} & 50 & 30 & 15 & 1 & Int. & 2749.8 \\
\hline \multicolumn{8}{|c|}{ Plackett-Burman Design - Replicate 5} & \multirow{3}{*}{ Y } \\
\hline \multirow[t]{2}{*}{$N^{\circ} \operatorname{Exp}$} & Rand & Duree_PDT & $\begin{array}{c}\text { End_Point_P } \\
\text { DT }\end{array}$ & $\begin{array}{c}\text { Periode_ } \\
\text { Signal }\end{array}$ & Intensité & $\begin{array}{l}\text { Periode_ } \\
\text { Inte }\end{array}$ & Bundle & \\
\hline & & $\mathrm{s}$ & $\%$ & $\mathrm{~s}$ & A & $\mathrm{s}$ & Spectro. & \\
\hline 6 & 1 & 1800 & 25 & 60 & 10 & 1 & Ext. & 3463 \\
\hline 4 & 2 & 1800 & 25 & 30 & 15 & 3 & Ext. & 5956 \\
\hline 8 & 3 & 900 & 25 & 30 & 10 & 1 & Int. & 4130.8 \\
\hline 5 & 4 & 900 & 50 & 30 & 10 & 3 & Ext. & 3471.4 \\
\hline 1 & 5 & 1800 & 50 & 60 & 10 & 3 & Int. & 3614.2 \\
\hline 2 & 6 & 900 & 50 & 60 & 15 & 1 & Ext. & 4779.3 \\
\hline 3 & 7 & 900 & 25 & 60 & 15 & 3 & Int. & 4462.8 \\
\hline 7 & 8 & 1800 & 50 & 30 & 15 & 1 & Int. & 3571.9 \\
\hline
\end{tabular}

Table 3: Description of the Design of Experiments based on a Plackett-Burman (Hadamard) matrix. The design was replicated five times in a randomized order of the experimental conditions. Each replicate corresponds to a different position of the optical fiber collecting the fluorescence spectra on the tissue. 


\begin{tabular}{c|cc|c}
\hline Experimental point & $P b_{\text {end }}(\%)$ & $T_{D}(\min )$ & Mean $Y(\%)$ \\
\hline \hline$(0,0)$ & 55 & 22.5 & 83.5 \\
\hline$(+\alpha, 0)$ & 80 & 22.5 & 49.5 \\
\hline$(+1,+1)$ & 73 & 27.8 & 46.5 \\
\hline$(0,+\alpha)$ & 55 & 30 & 79.3 \\
\hline$(-1,+1)$ & 37 & 27.8 & 82.7 \\
\hline$(-\alpha, 0)$ & 30 & 22.5 & 39.7 \\
\hline$(-1,-1)$ & 37 & 17.2 & 81.8 \\
\hline$(0,-\alpha)$ & 55 & 15 & 75.7 \\
\hline$(+1,-1)$ & 73 & 17.2 & 30.7 \\
\hline
\end{tabular}

Table 4: Experimental points coordinates and the corresponding mean fitting coefficients $(\mathrm{n}=3)$ 


\begin{tabular}{lllll}
\hline & Estimate & Std. Error & t-value & $\operatorname{Pr}(>|t|)$ \\
\hline \hline$b_{0}$ & -150.99 & 22.98 & -6.57 & $1.82 \mathrm{E}-07$ \\
$b_{1}$ & 26.59 & 17.37 & 1.53 & 0.135 \\
$b_{2}$ & 145.19 & 17.37 & 8.36 & $1.18 \mathrm{E}-09$ \\
$b_{3}$ & 7.41 & 17.37 & 0.427 & 0.672 \\
$b_{4}$ & -0.759 & 17.37 & -0.044 & 0.965 \\
$b_{5}$ & -24.57 & 17.37 & -1.415 & 0.167 \\
$b_{6}$ & 7.57 & 17.37 & 0.436 & 0.666 \\
\hline
\end{tabular}

Table 5: Results of the ANOVA analysis. 


\begin{tabular}{lllll}
\hline & Estimate & Std. Error & $\mathrm{t}$ value & $\operatorname{Pr}(>|t|)$ \\
\hline \hline$b_{0}$ & -216.78069 & 285.523673 & -0.7592 & 0.45615 \\
$b_{1}$ & 8.210335 & 4.408906 & 1.8622 & 0.07663 \\
$b_{2}$ & 8.728852 & 18.216433 & 0.4792 & 0.63677 \\
$b_{12}$ & -0.037718 & 0.097562 & -0.3866 & 0.70293 \\
$b_{11}$ & -0.074034 & 0.034598 & -2.1399 & 0.04426 \\
$b_{22}$ & -0.15181 & 0.385864 & -0.3934 & 0.69797 \\
\hline
\end{tabular}

Table 6: Results of the parameter estimation applied to the response surface model. 


\begin{tabular}{c|c|c}
\hline Parameter & Open-loop & Closed-loop \\
\hline \hline$\mu_{Y}(\%)$ & 71.3 & 83.3 \\
\hline$\sigma_{Y}(\%)$ & 23.4 & 2.5 \\
\hline
\end{tabular}

Table 7: Main statistics (average and standard deviation) of the fitting coefficients $Y$ for open- (without controller) and closed-loop (with controller) modes. 